\title{
Contemplating for the post pandemic era
}

\author{
Haemi Jee (D) https://orcid.org/0000-0002-9066-1472
}

Coronavirus disease 2019 (COVID-19) has been the most important issue for everyone in 2020 and will be throughout the upcoming new year. Unprecedented financial investments and collaborated efforts by leading scientists have been intensively focused on the search for an effective vaccine all over the world since the outbreak. According to the World Health Organization, there are more than 100 COVID-19 vaccine candidates with several successful reports on the outcome of the third phase of the clinical trial. Such reports have provided a hope for what it seemed like an endlessly long and dark tunnel. Although, at least several months will be needed for the novice vaccine to be effectively utilized to relieve of the pandemic situation, preparation for the post corona era does not seem to be impetuous.

Looking back at the history of the mankind, global outbreaks of lethal pathogens have been rather periodic. Even before the history began, outbreaks occurred. For example, Circa in 3000 B.C., plaque of Athens in 430 B.C., Black death in the 1300s, American plagues in 1500s, Spanish flue in 1900s, and Cholera in the 1800s, AIDS in 1980s, and Middle East respiratory syndrome in 2012 are among the many. Before the globalizations, these infections were epidemic, localized to a limited number of countries or regions and the outbreak intervals were comparatively sparse. Now the infections are becoming more pandemic and common due to evermore increasing mobility and accessibility to novice lands. COVID-like infections are possible in upcoming years. It would be the role of the scientists to prepare for the upcoming pathogens to minimize damage.

Despite continual efforts by numerous scientists to develop antiviral drugs to defend against viral infections, the effectiveness of the currently available antiviral drugs is limited in terms of types of virus and efficacy. Most of the antiviral drugs are nucleoside an- alogues to halt and inactivate DNA synthesis of a limited number of viral types with limited potency. Until virtually omnipotent antiviral drugs are discovered, the measures to protect against virus are limited to physically blocking infective agents by using masks, disinfectants, and social distancing from one another. In addition, strengthening one's immune system is the best defense against the bodily invasions.

There are a lot to be done by the rehabilitation experts. The experts should derive more effective methods to strengthen the immune system to defend against virus attack for individuals of different needs. In addition, the rehabilitation experts should focus on the types of damages done by the virus for more effective rehabilitation measures. Heart, lung, and brain damages may be evoked for increased heart failure, breathing problems, stroke as sequelae of COVID. Risk for neurological complications such as Parkinson disease and Alzheimer disease may also be increased along with many unknown long-term side effects.

To prevent and fight against short- and long-term complications, effective preventative and rehabilitation programs should be developed. Although another pandemic outbreak is unthinkable, exhaustive preparatory measures for all possible situations should be considered during the post pandemic era.

\section{CONFLICT OF INTEREST}

No potential conflict of interest relevant to this article was reported.

$$
\begin{array}{r}
\text { Department of Physical Therapy, Namseoul University, } 91 \text { Daehak-ro, } \\
\text { Seonghwan-eup, Sebuk-gu, Cheonan 31020, Korea } \\
\text { E-mail: amyjee@nsu.ac.kr }
\end{array}
$$

